\title{
Prediction of Transmitted and Received Power of Cellular Devices in an Indoor Femtocell Environment
}

\author{
D. Plets, S. Aerts, W. Joseph, L. Martens \\ Information Technology Department, Ghent University/iMinds \\ Gaston Crommenlaan 8, B-9050 Ghent, Belgium \\ david.plets@intec.ugent.be
}

\begin{abstract}
A prediction tool for the transmitted and received power of cellular devices in indoor femtocell environments is presented. Measurements in an office building are performed and compared with the predicted values. An excellent correspondence is obtained, with maximal errors around $3 \mathrm{~dB}$. This research allows predicting exposure doses in indoor femtocell scenarios.
\end{abstract}

\section{INTRODUCTION}

The need for increasing wireless data rates and the concern about possible adverse health effects of radio-frequency (RF) sources have started a trend towards an exposure-aware network planning. Femtocells, low-power cellular (indoor) base stations, not only improve connectivity, but are also assumed to reduce human exposure to electromagnetic waves. Although this is true for exposure caused by uplink traffic, the exposure due to downlink traffic may increase when using femtocells. Therefore, it is important to investigate and characterize both the power received and transmitted by the receiver device. In this paper, a prediction tool for the transmitted and received power of a cellular device in an indoor femtocell environment will be presented. The prediction will be compared with results from actual measurements in an office building. An accurate prediction allows calculating total (uplink and downlink) exposure doses, while often, only exposure due to the downlink signal is considered.

\section{Measurement Methodology}

The measurements are performed in the office building of Fig. 1 (90 m x $17 \mathrm{~m}$ ). At 50 locations, the Received Signal Strength Indication (RSSI) and the transmitted uplink power (TX) levels were monitored on a Nokia N95 8GB (Espoo, Finland), equipped with a Field Test Display (FTD) program. The 28 locations in the corridor with the femtocell are lineof-sight (LoS), the other 22 are Non Line-of-Sight (NLoS). The measurements were performed during a $3 \mathrm{G}$ phone call (25\% voice), connected to a femtocell base station (FBS) (type ePico3801B, Huawei, Shenzhen, Guangdong, China) at 2151.6 MHz (DL) and 1957.6 MHz (UL). The location of the femtocell is marked with a purple hexagon on Fig. 1, its Equivlant Isotropically Radiated Power (EIRP, $10 \mathrm{dBm}$ ) is indicated inside the hexagon. During each of the 50 measurements, the mobile was held horizontally at a height of $1.3 \mathrm{~m}$ above the floor and the RSSI, the maximum $\left(\mathrm{Tx}_{\max }\right)$ and the minimum
$\left(\mathrm{Tx}_{\min }\right)$ uplink power levels were recorded for four different orientations (90 degrees apart). In order to reduce the influence of the user's position and the mobile antenna directivity [1], the four RSSI, $\mathrm{Tx}_{\max }$, and $\mathrm{Tx}_{\min }$ values are averaged. $\mathrm{Tx}_{\mathrm{avg}}$ is defined as the average of $\mathrm{Tx}_{\max }$ and $\mathrm{Tx}_{\min }$. The RSSI values are assumed to correspond with the actual received power. Fig. 1 shows that more measurements are performed close to the femtocell, because of the more rapid variation of the device uplink and downlink power there. The transmitted and received powers will also be predicted by the WiCa Heuristic Indoor Propagation Prediction (WHIPP) tool [2]. It is developed for path loss and throughput calculations in indoor environments and is based on the dominant path model, i.e., the path along which the signal encounters the lowest obstruction between transmitter and receiver. The prediction model has been extensively validated with measurements in different building types, without any tuning of the model parameters. Tx $x_{\text {pred }}$ and $\mathrm{RSSI}_{\text {pred }}$ are defined as the predicted transmitted and received power respectively. $\mathrm{Tx}_{\text {pred }}$ is obtained by adding the predicted device-to-FBS path loss PL to the femtocell receiver sensitivity: for power saving purposes, the device will try to reduce its transmit power, based on the connection quality. We assume the femtocell sensitivity to be equal to $-110 \mathrm{dBm}$. $\mathrm{RSSI}_{\text {pred }}$ is obtained by subtracting PL from the EIRP.

\section{RESULTS}

Fig. 2 shows the measured and predicted device TX uplink power at the different measurement locations. The predicted power is limited at $-57 \mathrm{dBm}$, the lower limit of all observed TX powers. The predicted TX power is situated between the maximum and minimum measured TX powers and agrees excellently with its average uplink power $\mathrm{Tx}_{\mathrm{avg}}$. Table I lists the average deviation $\delta_{\text {avg }}$ between the predicted TX power $\mathrm{Tx}_{\mathrm{pred}}$ and the measured average $\mathrm{TX}$ power $\mathrm{Tx}_{\mathrm{avg}}$, as well as the corresponding standard deviation $\sigma_{\delta}$. Also average absolute deviations $|\delta|_{\text {avg }}$ and the corresponding standard deviation $\sigma_{|\delta|}$ are listed. All deviations are below $3 \mathrm{~dB}$, with limited standard deviation values. As an indication of the benefits of using a femtocell, Fig. 2 also shows the TX uplink power Txavg macrocell when connecting to an (outdoor) macrocell. Although the value depends on the building materials and the base station distance, it shows that the use of a femtocell leads to a 


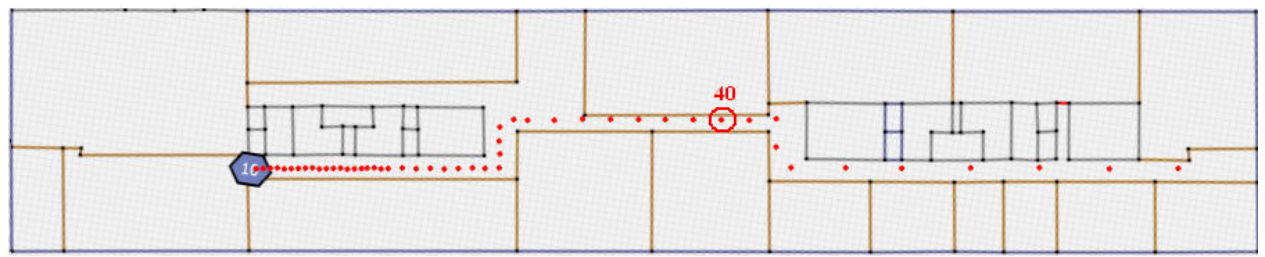

Fig. 1. Ground plan of office building $(90 \mathrm{~m} \mathrm{x} 17 \mathrm{~m}$ ) with indication of measurement locations (red dots), femtocell location (purple hexagon, EIRP = $10 \mathrm{dBm})$, and measurement number 40 .

lower exposure due to uplink traffic (higher uplink power for macrocell than for femtocell scenario).

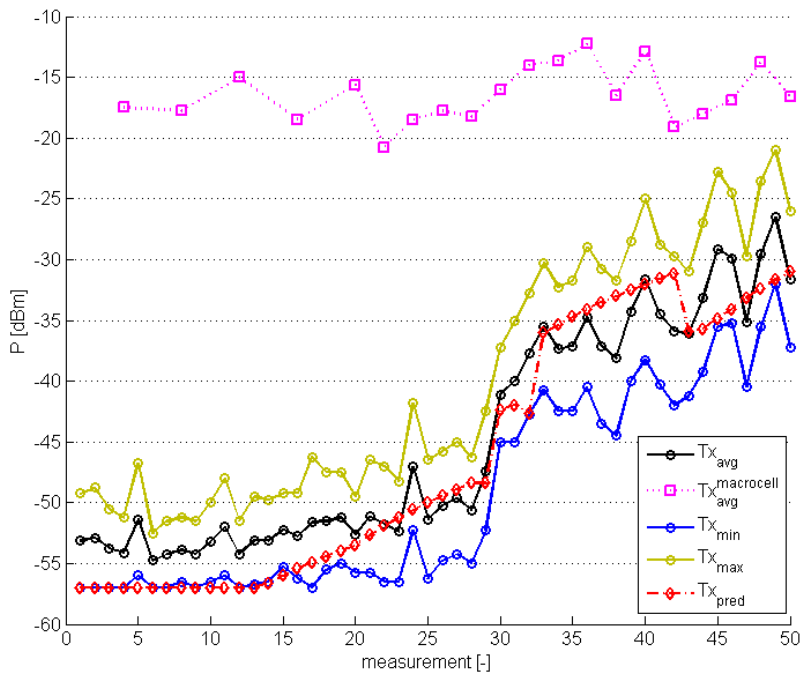

Fig. 2. Measured and predicted TX uplink power $\mathrm{P}$ as a function of measurement number (increasing measurement number corresponds with increasing distance to femtocell (see Fig. 1))

\begin{tabular}{|r||r|r|r|r||r|r|r|r|}
\hline \multicolumn{1}{|c||}{} & \multicolumn{4}{c|}{ RSSI (DL) } & \multicolumn{4}{c|}{ TX (UL) } \\
\hline & $\delta_{\text {avg }}$ & $\sigma_{\delta}$ & $|\delta|_{\text {avg }}$ & $\sigma_{|\delta|}$ & $\delta_{\text {avg }}$ & $\sigma_{\delta}$ & $|\delta|_{\text {avg }}$ & $\sigma_{|\delta|}$ \\
\hline \hline LoS & -3.0 & 3.8 & 4.1 & 2.6 & 2.3 & 2.0 & 2.8 & 1.3 \\
\hline NLoS & 0.3 & 2.3 & 1.4 & 1.1 & 0.2 & 3.2 & 2.6 & 1.7 \\
\hline All & -1.6 & 3.6 & 3.1 & 2.4 & 1.4 & 2.8 & 2.7 & 1.5 \\
\hline
\end{tabular}

TABLE I

AVERAGE AND AVERAGE ABSOLUTE DEVIATIONS BETWEEN PREDICTED AND MEASURED TRANSMITTED POWER (COLUMN 'TX') AND RECEIVED POWER (COLUMN 'RSSI'), AND CORRESPONDING STANDARD DEVIATIONS.

Fig. 3 shows the measured and predicted received power values (RSSI) at the different measurement locations. The RSSI is limited at $-51 \mathrm{dBm}$, the upper limit of all observed power values.

Table I lists the average deviation $\delta_{\text {avg }}$ between $\mathrm{RSSI}_{\text {pred }}$ and the measured average RSSI RSSI avg. Fig. 3 and Table I show that, again, very low prediction errors are obtained, especially in NLoS situations. Fig. 3 also shows the received power RSSI mavrocell when connecting to a macrocell. It shows that, when the user remains near the femtocell (measurement numbers $<40$, see Fig. 1) the use of a femtocell improves the connection quality, but also increases exposure due to the presence of the femtocell.

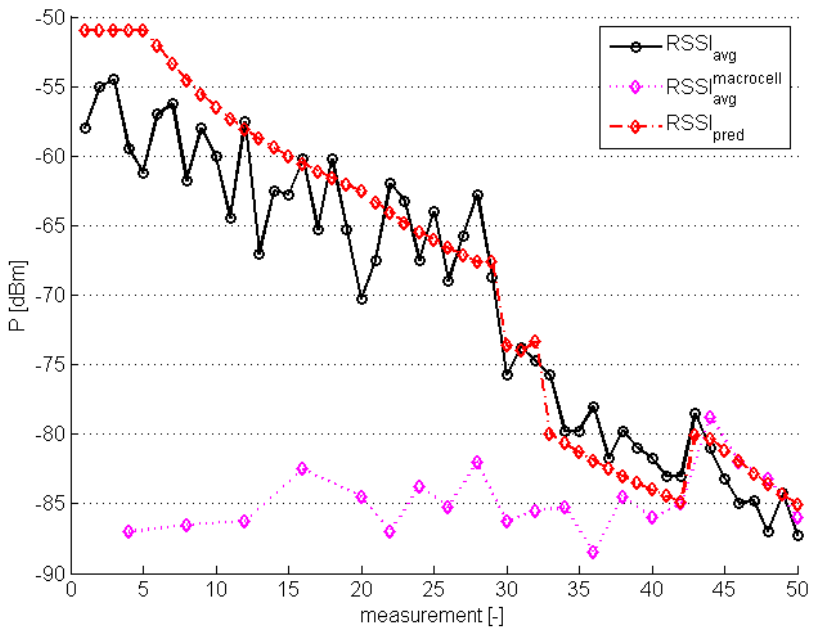

Fig. 3. Measured and predicted received power $\mathrm{P}$ as a function of measurement number.

\section{Conclusions}

A prediction tool for received and transmitted power of cellular devices in femtocell environments is presented. Excellent predictions are obtained, with average absolute prediction errors mostly below $3 \mathrm{~dB}$. Knowledge of downlink and uplink power allows calculating total exposure doses in different femtocell scenarios. These predictions can be used to perform exposure-aware network planning.

\section{ACKNOWLEDGEMENT}

This work has been carried out with the financial support of the iMinds-RAILS project. W. Joseph is a Post-Doctoral Fellow of the FWO-V (Research Foundation-Flanders).

\section{REFERENCES}

[1] A. Boursianis, P. Vanias, and T. Samaras, "Measurements for assessing the exposure from 3G femtocells," Radiation Protection Dosimetry), vol. 150, no. 2, pp. 158 - 167, 2012.

[2] D. Plets, W. Joseph, K. Vanhecke, E. Tanghe, and L. Martens, "Coverage Prediction and Optimization Algorithms for Indoor Environments," EURASIP Journal on Wireless Communications and Networking, Special Issue on Radio Propagation, Channel Modeling, and Wireless, Channel Simulation Tools for Heterogeneous Networking Evaluation, vol. 1, 2012. [Online]. Available: http://jwcn.eurasipjournals.com/content/2012/1/123 